\title{
CCDs on the Curtis Schmidt Telescope
}

\author{
Patrick Seitzer, Nancy Houk \\ Department of Astronomy, University of Michigan, 830 Dennison \\ Building, Ann Arbor, MI 48109-1090 USA
}

\begin{abstract}
The University of Michigan's Curtis Schmidt Telescope at CTIO has recently been equipped for CCD imaging at the Newtonian focus. A review is presented of the current status of this telescope, future upgrades, and some of the science being conducted.
\end{abstract}

\section{Introduction}

The University of Michigan's Heber D. Curtis Schmidt telescope was originally installed at the Portage Lake Observatory near Dexter, Michigan, in 1950. In 1966 this telescope was moved to the considerably more favorable skies at Cerro Tololo Inter-American Observatory, Chile, where one third of the time is made available to astronomers from the University of Michigan. Here it has been used in a photographic mode for several very significant survey projects (for example, see the review by Houk in this volume). The recent availability of large format CCDs has made them the most popular mode of observing on this telescope.

\section{Technical Details}

The Curtis Schmidt telescope has a clear aperture of $0.61 \mathrm{~m}$, a mirror diameter of $0.91 \mathrm{~m}$, a focal ratio of 3.5 , and a scale of $96.6 " / \mathrm{mm}$. This telescope is a close duplicate of the Burrell Schmidt telescope of the Case Western Reserve University now located on Kitt Peak.

For CCD imaging the CCD dewar, filter bolt, and shutter are all mounted outside the telescope tube at the Newtonian focus. Although this introduces an extra reflection and results in a smaller field of view than mounting the CCDs at the prime focus, as the Kiso group has done on their Schmidt, it has the advantages of being technically very simple to do and leaving the plateholder assembly intact for photographic work. It only requires about 10 minutes to position the Newtonian flat for CCD observations.

Currently only a 2 inch square filter bolt is available, and thus the unvignetted field of view is limited to just over 40 arcmin. But by the end of 1994 it is planned to construct in the shop at Michigan for installation at CTIO a filter bolt capable of holding 4 inch square filters. Along with a new shutter this should permit an unvignetted field of view approaching 1 degree in diameter.

The standard CCD detector is a front side illuminated Thomson $1024 \times 1024$ device. With 19 micron pixels, this device has a scale of 1.835 "/pixel and a field of view of $31^{\prime} \times 31^{\prime}$. It is coated to provide response in the $U$ and $B$ passbands. 
Later this year CTIO will install a STIS $2048 \times 2048$ CCD with 2.03 "pixels and a field of view of $1.1 \times 1.1$ degrees. All CCDs on this telescope are controlled by CTIO's new ARCON controller which has the capability of reading all four quadrants of a suitable CCD simultaneously. In such a fashion the Thomson device can be read out in less than 15 seconds.

The existing Newtonian mirror and support assembly were originally supplied for visual use of the telescope with eyepieces. Thus this focus may not be optimum for CCD imaging. We are currently investigating what the largest field of view available at any Newtonian focus would be, subject to the constraint that any flat not obstruct the beam any more than the current photographic plate holder. By moving the camera and Newtonian flat further up the tube from the primary and placing the focal plane as close as possible to the tube, it will be possible to increase the field of view significantly beyond the current 1 degree. We are also actively seeking to install a CCD with a smaller pixel size to better sample the stellar point spread function. With a larger diameter field and smaller pixel CCDs a field flattener will be necessary to remove the now significant effects of the curved focal plane.

\section{Scientific Projects}

A small CCD equipped telescope like the Curtis Schmidt is ideal for a number of projects which do not require reaching extremely faint limiting stellar magnitudes. Our experience has been that in a $600 \mathrm{~s} \mathrm{~V}$ band exposure, simple aperture photometry can reach $V=20$ with a precision of $0.1 \mathrm{mag}$ under dark sky conditions. And because of the fast $f /$ ratio of the Schmidt and high quantum efficiency of a CCD, very faint limiting surface brightness levels approaching 30 magnitudes per square arcsecond can be reached in total exposure times of a few hours (see the review by Armandroff in this volume). Here are a few of the CCD projects currently being undertaken by Michigan staff:

\subsection{Photometry of Clusters of Galaxies}

Rachel Pildis and Joel Bregman are obtaining mosaiced, multicolor images of clusters of galaxies for comparison with satellite X-ray observations. Deep BVI images will allow them to determine if these poor clusters have diffuse optical light (or to set stringent upper limits on such light). These results can then be compared to the detection or upper limits on diffuse $\mathrm{X}$-ray light in the clusters.

\subsection{Studies of Comets and Minor Planets}

The wide field of the Schmidt and speed of the CCD detectors makes precise astrometric and photometric studies of fast moving objects relatively easy. Michigan staff have obtained images of numerous recently discovered minor planets and have determined accurate astrometric positions tied to the HST Guide Star Catalog. During 1994 numerous observations will be obtained of Comet Shoemaker-Levy 9 as it approaches Jupiter. 


\subsection{Galactic Structure}

Seitzer and Bruce Carney (University of North Carolina) have been using UBVI band CCD images to study the distribution of stars in regions where the Galactic warp is predicted to be from HI observations. Follow up spectroscopy of stars in the V magnitude range 15 to 18 is being undertaken with the CTIO $4 \mathrm{~m}$ to confirm stars that may be part of the distant Galactic disk.

\subsection{Surface Photometry of Globular Clusters}

A survey is underway of $U$ band observations of Galactic globular clusters to determine structural parameters, in particular to establish the possible existence of statistically significant higher order structural moments beyond ellipticity. Observations in the $U$ band suppress the contributions of bright giants, and the current field of view is large enough to cover all but the largest globulars.

\section{Acknowledgements}

Thanks are due first to Victor Blanco, for his strong support of the Schmidt over the years, and for many helpful discussions. Many people at CTIO have made major contributions towards putting the CCD camera on the Schmidt despite an extremely limited budget. Among others, we wish to thank Bill Weller, Alistair Walker, Oscar Saa, Gabriel Perez, Chris Smith, Roger Smith, and Dan Smith for all of their efforts. 